\title{
Behavioural adaptations in egg laying ancestors facilitate evolutionary transitions to live birth
}

Amanda K. Pettersen ${ }^{1 *}$, Nathalie Feiner ${ }^{1}$, Daniel W.A. Noble ${ }^{2}$, Geoffrey M. While ${ }^{3}$, Charlie

K. Cornwallis ${ }^{1 \#} \&$ Tobias Uller ${ }^{1 \#}$

1. Department of Biology, Lund University, Lund, 22 362, Sweden

2. Division of Ecology and Evolution, Research School of Biology, The Australian National University, Canberra, 2600, Australia

3. School of Natural Sciences, University of Tasmania, Hobart, 7005, Australia

*Corresponding author

\#Joint senior author

Keywords: Viviparity, Oviparity, Thermal plasticity, Behavioural plasticity, Reproductive mode, Squamates, Lizards, Snakes 


\section{Abstract}

2 Live birth is a key innovation that has evolved from egg laying over 100 times in reptiles.

3 One significant feature of this transition is the thermal conditions experienced by developing

4 embryos. Egg-laying lizards and snakes often have preferred body temperatures that are

5 lethal to developing embryos that should prevent egg retention: how has viviparity repeatedly

6 evolved in the face of this pervasive mismatch? We resolve this paradox by conducting

7 phylogenetic analyses of adult and embryo thermal preferences across 224 species. Thermal

8 mismatches between mothers and offspring are widespread but resolved by gravid females

9 behaviourally down-regulating their body temperature towards the thermal optimum of

10 embryos. Importantly, this thermoregulatory behaviour evolved in ancestral egg-laying

11 species before the evolution of live birth. Maternal thermoregulatory behaviour therefore

12 bypasses the constraints imposed by a slowly evolving thermal physiology and is likely to

13 have been a key requirement for repeated transitions to live birth. 


\section{Introduction}

15 The evolution of live bearing is an important life-history transition in vertebrates ${ }^{1-3}$. The

16 ecological conditions that favour the transition from egg laying (oviparity) to live birth

17 (viviparity) are relatively well understood, especially in reptiles, with particularly strong

18 support for the adaptive value of viviparity in cool climates ${ }^{3,4}$. By retaining embryos

19 throughout development, mothers can buffer offspring from suboptimal nest temperatures,

20 thereby ensuring higher hatching success and increased offspring viability ${ }^{4}$. Live bearing also

21 allows females to maintain offspring at higher temperatures than those of potential nest sites,

22 effectively increasing developmental rates to facilitate early-season hatching ${ }^{5,6}$. This

23 transition has allowed reptile species to diversify and persist in cool climates globally ${ }^{7}$.

24 Despite its adaptive advantage, how viviparity initially evolved is unclear. A major

25 challenge to the evolution of viviparity is that egg-laying adult lizards and snakes typically

26 have preferred body temperatures that exceed the upper lethal limit of embryos ${ }^{8}$. For

27 example, the average nest temperature for the Iberian emerald lizard, Lacerta schreiberi, is

$2824^{\circ} \mathrm{C}$, rarely exceeding $30^{\circ} \mathrm{C}$, while the preferred body temperature of females is $33^{\circ} \mathrm{C}^{9}$.

29 Since embryos are well adapted to the temperatures they typically experience in the nest, they

30 generally have limited capacity to sustain development at temperatures outside a narrow

31 thermal window ${ }^{10,11}$. Prolonged exposure to temperatures beyond this range can result in

32 offspring abnormalities and even death ${ }^{12}$. A mismatch between thermal optima of embryos

33 and females should therefore prevent mothers from retaining embryos throughout

34 development, inhibiting evolutionary transitions to live birth $^{8}$.

35 Live birth has nevertheless evolved over 100 times in squamate reptiles ${ }^{13,14}$. How can

36 the repeated evolution of viviparity be reconciled with the widespread thermal mismatch

37 between embryos and adults in oviparous species? There are two potential scenarios by

38 which viviparity can evolve in the presence of maternal-embryo thermal mismatches. First, 
39 viviparity may only evolve from oviparity in species where there is no mismatch, that is,

40 where adult body temperature $\left(P_{b t}\right)$ and embryo thermal optima $\left(T_{\text {opt }}\right)$ are well aligned.

41 Second, females may behaviourally adjust their body temperature when pregnant to close the

42 gap between adult and embryo thermal optima, even when substantial mismatches in thermal

43 preferences exist. Such plasticity may come at a cost to female performance, but temporarily

44 shifting body temperature while gravid to match the thermal optima of embryos could

45 eliminate thermal barriers to the evolution of viviparity.

46 Explaining how the mismatch between female and offspring thermal optima have

47 been resolved in viviparous lineages is challenging. Adult and embryo thermal preferences of

48 the oviparous ancestors of viviparous lineages need to be reconstructed using available data

49 on extant species. In addition, data on thermal preferences across life stages are difficult to

50 obtain. Often only some measures (preferred body temperature of oviparous adults, optimal

51 incubation temperature of embryos, plasticity in female thermoregulation) are available for a

52 subset of species, leading to patchily distributed data (Figure 1). Recent advances in

53 phylogenetic methods help to resolve such issues, now making it possible to infer ancestral

54 states across multiple traits where information is non-overlapping across species (e.g., values

55 of some traits are only available for some species and vice versa). The accuracy of such

56 analyses are, however, reliant on the amount of variation in traits explained by phylogeny

57 (phylogenetic signal) as this determines the accuracy with which missing values can be

58 predicted $^{15}$.

59 We collated data on the thermal preferences of adult females (163 species) and

60 embryos (51 species) of squamate reptiles and combined this with data on their reproductive

61 mode (4162 species). Prior to running analyses, we estimated the phylogenetic signal in all

62 traits to ensure it was possible to infer ancestral values of traits given data limitations. We

63 found that there were high levels of phylogenetic signal in all traits (Tables S2, S6 \& S8). We 
64 further tested if models could accurately predict ancestral states using limited data by

65 restricting data on reproductive mode to just those species that also had thermal data (224

66 species). These analyses showed that it was possible to predict the reproductive modes of

674162 species and their ancestral nodes with $94 \%$ accuracy from data on just these 224 species

68 (Figure 3, Table S13).

69 Given phylogenetic models could accurately predict missing values and ancestral

70 states we proceeded with the following analyses: First, the ancestral states of preferred body

71 temperature of females and embryos were estimated to quantify the thermal conflict between

72 mothers and embryos at the origins of viviparity. Second, we tested the prediction that

73 transitions to viviparity have been restricted to oviparous lineages with a low degree of

74 maternal-embryo mismatch. This was assessed by calculating if viviparity has originated

75 more frequently from oviparous ancestors where female Pbt overlaps with the thermal optima

76 of embryos. Finally, we examined if females adjust their body temperature when gravid to

77 reduce the mismatch between adult and embryo thermal optima (52 species), and if so,

78 whether this behaviour evolved prior to the emergence of viviparity, or afterwards in

79 response to such mismatch. 


\section{Results and Discussion}

81 Wide-spread mismatch in thermal optima of oviparous mothers and their embryos

82 Across oviparous lizards and snakes, there were many species with a significant disparity

83 between adult female preferred body temperature $\left(P_{b t}\right)$ and the temperature that maximizes

84 hatching success $\left(T_{\text {opt }}\right)$ (Multi-response BPMM (MR-BPMM): posterior mode (PM) of

85 difference $=4.42,95 \%$ Credible Interval $(\mathrm{CI}):-2.98,11.53, p M C M C=0.12$. Figure $1 \& 2$;

86 Table S3). The female $P_{b t}$ of oviparous species is on average $5^{\circ} \mathrm{C}$ higher than $T_{\text {opt }}$ (raw data

87 mean \pm SD $P_{b t} 103$ species: $32.41 \pm 4.15^{\circ} \mathrm{C} ; T_{o p t} 47$ species: $\left.27.15 \pm 2.12{ }^{\circ} \mathrm{C}\right)$. Incubation

88 experiments have shown that such a difference can significantly reduce hatching success,

89 illustrating that exposure to those thermal conditions throughout development via the

90 retention of offspring may jeopardise embryo survival (reviewed in $\left.{ }^{16}\right) . P_{b t}$ and $T_{o p t}$ of

91 oviparous species were both estimated to evolve slowly (Figure 1; MR-BPMM: $P_{b t}$

92 phylogenetic signal (phylo $H^{2}$ ): 0.94 , CI: 0.84, 0.99. $T_{\text {opt }}$ phylo $H^{2}: 0.94$, CI: $0.78,0.99$. Table

$93 \mathrm{~S} 3$ ) and female $P_{b t}$ and offspring $T_{\text {opt }}$ only show weak evidence of coevolving (Figure 2;

94 phylogenetic correlation (MR-BPMM): $\mathrm{PM}=0.69$, CI: $-0.22,0.90, p M C M C=0.21$. Table

95 S4), suggesting that this mismatch may be difficult to resolve.

97 Transitions to live birth occur despite mismatches between females and embryos

98 We found evidence that preferred body temperature was lower in viviparous, compared with

99 oviparous, lineages (MR-BPMM: $\mathrm{PM}=-0.27$, CI: $-0.49,-0.01, p M C M C=0.03$; Figure 3;

100 Table S6). This is consistent with the hypothesis that viviparity is an adaptation to cool

101 climates ${ }^{2,3,17}$. However, reconstructions of ancestral preferred body temperatures of adults

102 indicated that in the majority of lineages, lower preferred body temperatures evolved after,

103 not before, transitions to live bearing. This was evident from the lack of difference in $P_{b t}$

104 between ancestors of egg-laying and live-bearing lineages (MR-BPMM, PM=0.07, CI: - 
$1051.80,1.20, p M C M C=0.41$; Table S7). Additionally, live-bearing lineages with oviparous

106 ancestors showed higher $P_{b t}$ than those with live-bearing ancestors (MR-BPMM, PM = -1.46,

107 CI: -2.79, 0.77, $p M C M C=0.18$; Table S7).

Ancestral reconstructions of $P b t$ and Topt of oviparous species showed that transitions

109 from egg laying to live bearing were no more likely to occur when adult and embryo thermal

110 optima were similar (proportion of lineages with thermal mismatches: ancestors of live

111 bearing species 69\%, ancestors of egg laying species 78\%. Table S5). Only eight of the

112 estimated 26 evolutionary origins of live birth had adult and embryo thermal optima that

113 overlapped, which was similar to the proportion of lineages where live bearing did not occur

114 (Table S5). In the remaining 18 egg-laying ancestors of live-bearing species, the thermal

115 optimum of embryos was significantly lower than the preferred body temperature of adults

116 (there were no cases of $T_{\text {opt }}$ being significantly higher than $P_{b t}$; Table S5). In summary, the

117 degree of similarity between adult $P_{b t}$ and embryo $T_{o p t}$ does not predict transitions towards

118 viviparity, and the lower $P_{b t}$ observed for viviparous taxa appears to have evolved after

119 transitions to live birth, not before.

121 Female behavioural plasticity resolves the constraints imposed by thermal physiology

122 Next, we tested the hypothesis that the thermal mismatch between females and embryos

123 during the evolution of viviparity is resolved by females adjusting their body temperature

124 when gravid to meet the thermal requirements of their embryos ${ }^{18}$. We collected data on the

125 preferred body temperature of gravid $\left(P_{b t-g}\right)$ and non-gravid $\left(P_{b t-n g}\right)$ adult females from 52

126 species of lizards and snakes from published field and experimental studies (viviparous $\mathrm{n}=$

12732 , oviparous $n=20$. Table S1). Using these data, an effect size of female behavioural

128 plasticity, Hedges' $g$ was calculated (positive values $=$ higher $P_{b t}$ when gravid, negative

129 values $=$ lower $P_{b t}$ when gravid). 
131 and the degree to which females reduced their body temperature when gravid (MR-BPMM:

$132 \mathrm{PM}=-0.38, \mathrm{CI}:-0.71,0.13, p M C M C=0.08$. Figure 4; Table S8). Females with high $P b t$

133 have therefore evolved to behaviourally reduce their body temperature when gravid to a

134 greater extent than females with low Pbt. This relationship was not restricted to viviparous

135 lineages, as would have been expected if female behavioural plasticity is an adaptation that

136 evolves in response to selection for embryo retention. Instead, comparable phylogenetic

137 correlations between Hedges' $g$ and $P b t$ were evident in both oviparous and viviparous taxa,

138 showing that behavioural adjustment of temperature when gravid occurs to a similar extent in

139 both egg-laying and live-bearing species (Egg laying: $\mathrm{PM}=-0.83, \mathrm{CI}:-0.98,-0.02, p M C M C$

$140=0.05$. Live bearing: $\mathrm{PM}=-0.90, \mathrm{CI}:-0.99,-0.25, p M C M C=0.02$. Table S9). Furthermore,

141 when we reconstructed the values of Hedges' $g$ (i.e., the effect of gravidity on body

142 temperature) for ancestors of egg-laying and live-bearing species, we found that thermal

143 mismatches between adults and embryos were resolved by females adjusting their body

144 temperature when gravid (Figure 4A; MR-BPMM: Egg-laying Hedges' $g$ PM = -0.71, CI = -

$1451.21,-0.30, p M C M C<0.01$. Live bearing Hedges' $g$ PM $=-0.74, \mathrm{CI}=-1.34,-0.31, p M C M C$

$146<0.01$. Table S10). As a result, Hedges' $g$ did not differ between the ancestors of oviparous

147 and viviparous species (Figure 4B; Table S9-S11), suggesting that behavioural plasticity was

148 likely present prior to the emergence of live birth.

149 Our results illustrate that an ancient and evolutionarily conserved ability of egg laying

150 females to adjust their body temperature during pregnancy provides a general resolution to

151 the conflict over adult and embryo thermal optima that paves the way for the evolution of live

152 bearing. The down-regulation of female body temperature in egg-laying species while gravid

153 may appear surprising considering that most of these species lay their eggs within the early

154 stages of development (commonly around the time of limb bud formation ${ }^{19}$ ). However, early 
155 developmental stages, involving gastrulation, neurulation and organogenesis, are potentially

156 even more sensitive to temperature stress than later stages, which are predominantly

157 associated with growth ${ }^{8,10}$. The temperature sensitivity of early-stage embryos therefore

158 suggests that resolutions to mother-offspring thermal conflicts are required in both egg-laying

159 and live-bearing species. If true, the key innovation of live birth may owe its evolutionary

160 origin to mechanisms of temperature regulation put in place long before live birth emerged.

161 We suggest that this behavioural flexibility of female lizards and snakes enabled the

162 gradual evolution of live birth by helping traverse potential fitness valleys associated with

163 embryo retention. In many live-bearing lineages, temperature mismatches have subsequently

164 been eliminated through the evolution of a lower preferred body temperature in females.

165 However, behavioural flexibility is frequently maintained in viviparous lineages that have

166 colonised cool climates. Such behavioural flexibility provides females with the means to

167 upregulate their thermoregulation in order to maintain embryos at temperatures significantly

168 warmer than the external environment ${ }^{2,5,6,20,21}$.

169 In conclusion, female thermoregulatory behaviour is a key adaptation that overcomes

170 the constraints imposed by thermal mismatches between mothers and embryos, and has

171 facilitated both the evolution of live birth, and expansion of reptiles into a variety of

172 environments. 


\section{Methods}

\section{Literature search and data collection}

175 To investigate the relationship between maternal behavioural plasticity and embryo thermal

176 sensitivity, we collected existing data on reproductive mode (oviparous versus viviparous),

177 the female preferred body temperature $\left(P_{b t}\right)$ (“" $P_{b t}$ dataset”), the temperature at which hatching

178 success was maximised ( $\left.T_{\text {opt }}\right)$ (“" $T_{\text {opt }}$ dataset”), and female body temperature when gravid $\left(P_{b t-}\right.$

$179 g$ ) and not gravid $\left(P_{b t-n g}\right)$ ("Hedges' $g$ dataset"). Complete data are presented in Table S1.

180 Data were collected for each of the variables from published literature. We conducted a

181 literature search using ISI Web of Science (v.5.30) with search terms specific to each dataset.

182 The $P_{b t}$ dataset provided preferred body temperatures $\left(P_{b t}\right)$ of adult females measured at

183 one point in time. We used the search terms 'body temperature*', along with one of the

184 following: 'squamat*', 'lizard*', 'snake*' which yielded a total of 1075 papers. We only

185 used data from studies where $P_{b t}$ for females was stated explicitly, unless pooled male/female

186 data stated no significant effect of sex. Data on females that were described to be gravid or

187 data collected during the reproductive season were excluded. This provided a final dataset of

188 female preferred body temperature for 163 species that was independent of the gravid and

189 non-gravid measures used to calculate Hedges' $g$ (live bearing: $n=61$ and egg laying: $n=$

190103 , note $P_{b t}$ data for Zootoca vivipara was available for both reproductive modes and both

191 were included in the analyses - see below).

192 The $T_{\text {opt }}$ dataset quantified the temperature that maximises embryonic survival (measured

193 as hatching success; $T_{\text {opt }}$ ) in 51 egg laying species (Figure S1). Hatching success data were

194 taken from the Reptile Developmental Database ${ }^{22}$ and supplemented with additional studies

195 using the same method described for the $P_{b t}$ dataset (search terms: "temperature* AND

196 incubat* AND hatch* OR surv*', along with one of the following: 'squamat*', 'lizard*',

197 'snake*'), yielding a total of 671 papers. We only included studies where three or more 
198 constant temperature treatments were used under controlled laboratory conditions, resulting

199 in 661 papers being rejected due to irrelevance or overlap with the Reptile Development

200 Database. This resulted in a final Topt dataset, consisting of 51 species, obtained from 81

201 studies. Given that $T_{\text {opt }}$ had a strong phylogenetic signal (Phylogenetic Heritability $\left(H^{2}\right)=$

202 91.93\%, 95\% CI: 71.65 - 98.16\%; Table S3) we fitted a Bayesian Phylogenetic Mixed

203 Effects Model (BPMM) to hatching success data for all species jointly. Including

204 phylogenetic information allowed for the $T_{o p t}$ of each species to be estimated with greater

205 accuracy and precision given that the range and number of temperatures across species varied

206 (range: $10-40^{\circ} \mathrm{C}$, mean number of temperatures per species $\pm \mathrm{SD}: 7.62 \pm 4.29$ ). Compared to

207 non-phylogenetic models, $T_{\text {opt }}$ estimates produced from phylogenetic models (BPMM)

208 showed smaller sampling error and avoided convergence problems in estimating model

209 parameters. Importantly, this approach was not used to estimate $T_{\text {opt }}$ data for species without

210 data, only to better predict $T_{o p t}$ values for species for which there were data. The $T_{o p t}$ BPMM

211 model was run for 1,100,000 iterations with a burn-in of 100,000 iterations and thinning rate

212 of 500, leaving us with 2,000 samples from the posterior distribution. Autocorrelation was

213 low (lag values $<0.1$ ) and trace plots showed chains mixed well for all parameters. Our

214 model included temperature as a fixed effect (estimating both a linear and quadratic slopes)

215 and random slopes of temperature (linear and quadratic slopes) fitted at the phylogenetic

216 level. From our BPMM we estimated $T_{\text {opt }}$, and its corresponding sampling variance, using the

217 posterior distribution of fixed effects and best linear unbiased predictors (BLUPs) for the

218 random slopes (linear and quadratic) for each species as follows:

$$
T o p t=-\frac{\left(T_{f}+T_{s p}\right)}{\left(2\left(T_{f}^{2}+T_{s p}^{2}\right)\right)}
$$

219 Where $T_{f}$ and $T_{f}^{2}$ are the posterior linear and quadratic fixed effect estimates for temperature 220 and $T_{s p}$ and $T_{s p}^{2}$ are the posterior BLUPs for a given species extracted from the phylogenetic 
221 random slopes. Calculating $T_{\text {opt }}$ using the posterior distribution of fixed and random effects

222 meant that sampling error for a given species could be propagated to subsequent analyses

223 (see below).

224 For the final "Hedges' $g$ " dataset, we collected mean, standard deviation and sample sizes

225 for female preferred body temperatures when gravid $\left(P_{b t-g}\right)$ and non-gravid $\left(P_{b t-n g}\right)$

226 (independent from the $P_{b t}$ dataset) from the same study/population. Within the 'title',

227 'abstract' or 'keywords' we used search terms 'body temperature* AND gravid* OR

228 reproduct*' along with one of the following: 'squamat*', 'lizard*', 'snake*' which yielded a

229 total of 721 papers. An effect size of female adjustment of body temperature when gravid for

230 each species was measured as the standardised mean differences (Hedges' $g$ ) in preferred

231 body temperatures between non-gravid and gravid females $\left(P_{b t-g}-P_{b t-n g}\right)$, adjusting for small

232 sample sizes ${ }^{23}$.

233 We included both field and laboratory measures of female body temperature, comprising

234 studies that directly compared $P_{b t-g}$ and $P_{b t-n g}$. Only studies that provided both sample size

235 and error around mean of preferred body temperature were included. Of the 721 papers 648

236 papers were excluded due to irrelevance or insufficient data. Combined, this yielded a total of

23773 studies published up to July 2019 from which effect sizes were calculated for 52 species

238 (live bearing: $\mathrm{n}=32$ and egg laying: $\mathrm{n}=20$ ). The majority of studies used artificial

239 temperature gradients in the laboratory $(n=37)$ or measured preferred basking temperature in

240 the field $(n=36)$. Laboratory studies generally measured body temperature in the same

241 female during gestation $\left(P_{b t-g}\right)$ and either before or after gestation $\left(P_{b t-n g}\right)$ as repeated

242 measures. In contrast, field studies often measured body temperature on a population during

243 the reproductive season, comparing body temperatures in gravid and non-gravid females at a

244 single time point. We examined the mean Hedges' $g$ between laboratory or field studies and 
245 found there were no significant differences $(\mathrm{PM}$ (Oviparous) $=-0.472, \mathrm{CI}=-1.685,0.557$;

$246 \mathrm{PM}$ (Viviparous) $=0.564, \mathrm{CI}=-0.250,1.313$. Table S14. See Verification analyses below).

247 The final full dataset (Table S1) contained a total of 224 squamate species. Due to the

248 inherent paucity of data, particularly for hatching success and body temperature in gravid

249 females, there was little overlap of species between datasets - we found 39 species had data

250 available for 2 datasets, while we were only able to obtain data for 4 species across all 3

251 datasets. Two species in our data are both oviparous and viviparous (Saiphos equalis and Z.

252 vivpara). While for $S$. equalis all data were from an oviparous population, for Z. vivipara

253 there were data for both oviparous and viviparous populations and we treated these different

254 reproductive modes as being distinct in our dataset ${ }^{24}$.

255

256 Quantification and Statistical Analysis

257 Prior to running our main analyses, we estimated the mode of evolution that best explained

258 how traits evolved using Phylogenetic Generalised Least Squares (PGLS). Brownian motion

259 was the best fitting model for all traits (Table S2). We then used Bayesian Phylogenetic

260 Mixed Effects Models with single (BPMM) and multiple response variables (MR-BPMM),

261 which enabled us to overcome the challenge of missing data across species, to estimate

262 phylogenetic correlations between traits and reconstruct ancestral states. The robustness of

263 ancestral reconstructions was examined using stochastic character mapping (SCM; Table

$264 \mathrm{~S} 12)$. All analyses were conducted in $\mathrm{R}$ version $4.0 .1^{25}$.

265

266 Assessing modes of evolution using Phylogenetic Generalised Least Squares (PGLS)

267 An assumption of many phylogenetic comparative methods is that traits evolve via a

268 Brownian motion process ${ }^{26}$. This assumption was assessed by estimating the mode of

269 evolution that best describes the phylogenetic distribution of reproductive mode, $P B T$ and 
270 Topt using PGLS implemented with R packages 'ape' and 'nlme ${ }^{, 27,28}$. This was done by

271 comparing PGLS models with different correlation structures constructed from the phylogeny

272 that corresponded to BM, Ornstein-Uhlenbeck (OU: corMartins in 'ape') and Accelerated

273 Decelerated (ACDC: corMartins in 'ape') modes of evolution. In OU models' traits undergo

274 a BM process but evolve towards a central tendency with a strength proportional to parameter

275 alpha $(\alpha)$, and in accelerated/decelerated models of evolution (ACDC) traits diverge under a

276 BM model, but rates of evolution accelerate or decelerate over time according to parameter $\mathrm{g}$

277 (Acceleration: $\mathrm{g}<1 . \mathrm{BM}: \mathrm{g}=1$. Deceleration $\mathrm{g}>1$ ). Estimating parameters, such as $\alpha$ and $\mathrm{g}$,

278 directly from the data can be unstable ${ }^{29,30}$. Therefore, we estimated values of $\alpha(\mathrm{OU})$ and

279 Bloomberg's g (ACDC) by fixing $\alpha$ and g at 0.1 increments within the range of 0 to 10 and

280 used AIC values to identify best-fit models. For BM models AIC values were taken from

281 models with an optimised value of lambda rather than a strict BM model (lambda = 1). For

282 all traits there was significant phylogenetic signature and BM best explained the way traits

283 have evolved (Table S2: R code: Section 0 ).

286 We implemented BPMMs in $R$ with the MCMCglmm package ${ }^{31}$. All traits $\left(T_{o p t}, P_{b b}\right.$, Hedges'

$287 g$ ) were modelled with Gaussian error distributions apart from the probability of viviparity

288 which was modelled using a binary error distribution with a logit link function. The sample

289 sizes for each species varied across traits, which was accounted for in models by weighting

290 each data point by their inverse sampling variances using the 'mev' term in MCMCglmm.

291 Samples sizes were not available for reproductive mode as this is measured without error due

292 to it being invariant within populations. Therefore, the mev term for reproductive mode was

293 specified as 0. 
295 for in two ways. In the main analyses (see specific analyses below for details) where multi-

296 response models were used to examine relationships between traits, a single data point was

297 included for each species. Therefore, a weighted mean for Hedges' $g$ for each species was

298 calculated. For the verification analyses of Hedges' $g$ where other traits were not included,

299 species was included as a random effect to account for multiple data points per species.

$300 \quad$ Phylogenetic relationships were modelled using a published phylogeny comprising

301 over 4000 species of squamates ${ }^{32}$ that was pruned to the 224 species in our dataset using the

302 ape package in $R^{27}$. All analyses were conducted on the trimmed tree (Figure 1), and

303 ancestral states were then verified using all available reproductive mode data (4162 species)

304 and the full tree - all results remained the same (See verification analyses. Table S13, Figure

305 S3). The random effect animal, with a covariance matrix derived from the phylogenetic tree

306 was included in all models ${ }^{31}$. We calculated the phylogenetic signature (equivalent to

307 heritability, $H^{2}$, in the terminology of $M C M C g l m m$ ) for each trait as the variance explained

308 by animal relative to total variance (animal and residual). In the case of binary variables

309 (reproductive mode) the residual variance is not identifiable and we therefore used the

310 intraclass coefficient of variation (ICC: phylogenetic variance / (phylogenetic variance +

311 residual variance $\left.+\mathrm{pi}^{2 / 3}\right) * 100$ ) to examine phylogenetic signature ${ }^{33}$. More traditional methods

312 were also used to validate estimates of phylogenetic signal. Pagel's lambda $(\lambda)$ from $p g l s$

313 models $^{34,35}$ and Blomberg's $\mathrm{K}(K)$ in phytools using the function phylosig ${ }^{36,37}$, which gave

314 comparable estimates to BPMMs (see R code section 0).

316 Multi-response BPMMs (MR-BPMMs) can also be fitted with MCMCglmm that allow the

317 phylogenetic and within-species (residual) correlations between traits to be estimated.

318 Correlations between traits (e.g., A \& B) were calculated as: 


$$
\frac{\operatorname{cov}(A, B)}{\sqrt{(\operatorname{var}(A) \cdot \operatorname{var}(B))}}
$$

Missing data across species

BPMMs allow missing data in response variables to be predicted, which was

322 particularly useful as values of traits were not available for all species $\left(T_{o p t}=51\right.$ species; $P_{b t}=$

323163 species; Hedges' $\mathrm{g}=52$ species). The accuracy with which missing data is predicted is

324 related to the phylogenetic signal in traits and the strength of phylogenetic correlations

325 between traits ${ }^{15}$. All traits had high phylogenetic signal (phylogenetic heritability $>80 \%$ )

326 producing high correspondence between raw and predicted values (Figure S2; See also

327 Verification analyses "2. Checking the ability of BPMMs to predict missing data”). As a

328 result, our BPMMs enabled us to deal with the fact that not all traits have been measured in

329 all species.

Imputation for missing sampling variances

332 The accuracy of measures of $T_{o p t}, P_{b t}$, and Hedges' $g$ varied across species due to study

333 design and sample sizes which can be accounted for by weighting data points by their inverse

334 sampling variance. However, missing values in sampling variances are not permitted in

$335 M C M C g l m m$. As data on the error and sample size was missing for $T_{o p t}, P_{b t}$ and Hedges' $g$ it

336 would not have been possible to account for sampling error in our analyses without

337 drastically reducing the size of our dataset. Consequently, we used multiple imputation with

338 predictive mean matching in the mice package in $\mathrm{R}$ to impute missing error and sample

$339 \operatorname{sizes}^{38}$. To incorporate uncertainty in imputations, 20 complete datasets were generated, and

340 all analyses were conducted by sampling across these datasets. Each model sampled through

341 the 20 datasets 75 times (1500 sampling events) for 2000 iterations with only the last iteration

342 being saved. Estimates from the last iteration of each sampling event $i$ were used as the 
343 starting parameter values for the next $i+1$. This led to a total posterior sample of 1500

344 iterations, the first 500 iterations were discarded as a burn-in and the remaining 1000 (50 per

345 dataset) were used to estimate parameters. Pooling of posterior distributions from model

346 parameters from across each of the $n=20$ datasets enabled imputation uncertainty in

347 sampling variances to be accounted for.

Model convergence, prior settings and characterisation of posterior distributions

350 Non-informative uniform priors were used for fixed effects and inverse-Wishart priors for

351 random effects $\left(V=1, \mathrm{nu}=0.002 ;{ }^{31}\right)$ apart from models with binary response variables (e.g.

352 reproductive mode). For binary variables the residual variance was fixed to 1 and we

353 specified a fixed effect prior that is relatively flat on the logit scale $(\mathrm{mu}=0, \mathrm{~V}=$ residual

354 variance $\left.\mathrm{x} \pi^{2 / 3}\right)$ and parameter-expanded random effect priors $(V=1, \mathrm{nu}=0.002$,

355 alpha.mu=0, alpha. $\mathrm{V}=1000$ ). To examine model convergence we ran three independent

356 MCMC chains and examined autocorrelation, which was low (lag values $<0.1$ ), trace plots,

357 which showed chains mixed well, and Gelman and Rubin's convergence diagnostic that

358 models converged (potential scale reduction factors were all below 1.1: $\mathrm{R}$ function

359 gelman.diag ${ }^{39}$. The convergence of models of the probability of viviparity (binary response

360 variable) occurred more slowly and therefore were run for longer (burn-in $=1000000$,

361 iterations $=6000000$, thinning $=5000$ ). All other models used the run settings specified in the

362 data imputation section. Posterior distributions of all parameters were characterised using

363 modes and 95\% credible intervals (CIs) throughout. Effects were regarded as significant

364 where CIs did not span 0. pMCMC (number of iterations above or below 0 / total number of

365 iterations) are also presented to facilitate general interpretation. 
368 We conducted six main analyses: 1) Phylogenetic signature in $P_{b t}$ and $T_{o p t}$ was estimated

369 using a MR-BPMM of $P_{b t}$ and $T_{o p t}$ with heterogenous phylogenetic and residual covariance

370 matrices fitted as random effects (R code model M1.1. Table S3). 2) The phylogenetic

371 correlation between $P_{b t}$ and $T_{o p t}$ was estimated using a MR-BPMM with unstructured

372 phylogenetic and residual covariance matrices fitted as random effects (R code model M1.4.

373 Table S4). 3) To quantify the number of ancestral egg-laying with and without mismatched

374 adult female and embryo thermal optima a two-step approach was used. The ancestral states

375 of reproductive mode were first estimated for each node in the phylogenetic tree using a

376 BPMM with the probability of live bearing as the response variable (R code model M3.0).

377 Next a MR-BPPM with $P_{b t}, T_{\text {opt }}$ and Hedges' $g$ as response variables was used (R code model

378 M3.1) to reconstruct ancestral states for each trait. From this model mismatches in thermal

379 optima (CI of $P_{b t}-T_{\text {opt }}$ not overlapping 0) and levels of female behavioural plasticity were

380 estimated (Table S5). Separate models were used for reproductive mode and $P_{b t}, T_{o p t}$ and

381 Hedges' $g$ to ensure that ancestral estimates were not influenced by the strong correlation

382 between live bearing and $P_{b t}$ in extant species. 4) The phylogenetic correlation between the

383 probability of live bearing and $P_{b t}$ was estimated using a MR-BPMM with unstructured

384 phylogenetic and residual covariance matrices fitted as random effects (R code model M2.1.

385 Table S6). 5) To examine if female plasticity differed between the ancestors of oviparous and

386 viviparous lineages with and without mismatched mother-offspring thermal optima, we

387 examined estimates of Hedges' $g$ in relation to predicted states of reproductive mode (R code

388 model M3.1. Table S7 \& S10-S11). 6) The phylogenetic correlation between $P_{b t}$ and Hedges'

$389 g$ was estimated across all species (R code model M3.1. Table S8), as well as for egg-laying

390 and live-bearing species separately using an MR-BPMM with separate unstructured

391 phylogenetic and residual covariance matrices for each reproductive mode ( $\mathrm{R}$ code model

392 M4.1. Table S9). 


\section{Verification analyses}

\section{Checking ancestral reconstructions with Stochastic character mapping (SCM)}

397 The robustness of ancestral state reconstructions of reproductive mode was examined by

398 assessing the correspondence between the estimates from MCMCglmm with those obtained

399 from SCM. The SCM approach calculates the conditional likelihood that each ancestral node

400 is oviparous or viviparous based on an estimated transition rate matrix (Q) between

401 reproductive modes and the length of the branch associated with that node. Based on these

402 conditional likelihoods, ancestral states at each node are stochastically simulated and used in

403 combination with observations at the tips to reconstruct a character history along each

404 branch. Each character history is simulated using a continuous-time Markov model where

405 changes between states and the time spent in each state is modelled as a Poisson process (see

$406{ }^{40}$ for details). We found extremely good correspondence between estimates of ancestral

407 states obtained from MCMCglmm models and SCM showing inferences of ancestral states

408 were robust to methodological details (Table S12).

409

\section{2. Checking the ability of BPMMs to predict missing data}

411 We examined how well models predicted missing values by running a BPMM of

412 reproductive mode on the full tree (4162 species) using only phenotypic data from the 224

413 species included in the main analyses (R code model M5.3: Table S13). The model settings

414 were identical to those described above for modelling reproductive mode. The accuracy of

415 the model predictions for tips in the tree were checked against raw data ${ }^{41}$, and model

416 predictions for tree nodes were checked against reconstructions of ancestral states obtained

417 from a BPMM using the full tree and full dataset (R code model M5.2. Table S13). 
419 3. Checking for differences in Hedges' $g$ between laboratory and field studies

420 Differences in the ability of laboratory versus field studies to estimate plasticity in female

421 body temperature when gravid was examined using a BPMM of Hedges' $g$ with study type as

422 a fixed effect (R code model M5.4. Table S14). 


\section{Data and code availability}

424 All data and code are publicly available at: https://doi.org/10.17605/OSF.IO/JT28V

425

426 Author Contributions: Conceptualization, N.F., C.K.C. and T.U.; Methodology, D.W.A.N.

427 and C.K.C.; Formal analysis, A.K.P., D.W.A.N. and C.K.C; Investigation, A.K.P.,

428 D.W.A.N., G.M.W. and T.U.; Data curation, A.K.P., D.W.A.N., C.K.C. and T.U.; Writing 429 original draft, A.K.P, N.F., C.K.C and T.U.; Writing - Reviewing \& Editing, A.K.P., N.F.,

430 D.W.A.N., G.M.W., C.K.C. and T.U.; Visualization, A.K.P., N.F., D.W.A.N. and C.K.C.;

431 Supervision, C.K.C. and T.U.; Funding acquisition, A.K.P. and T.U.

432

433 Competing Interest Statement: The authors declare no competing interests. 


\section{References}

435 1. Doody, J. S. \& Moore, J. A. Conceptual model for thermal limits on the distribution of 436 reptiles. Herpetological Conservation and Biology 5, 283-289 (2010).

437 2. Shine, R. The evolution of viviparity in reptiles: An ecological analysis. in Biology of the 438 Reptilia vol. 15 605-694 (John Wiley \& Sons, Ltd, 1985).

439 3. Lambert, S. M. \& Wiens, J. J. Evolution of viviparity: a phylogenetic test of the cold-

440 climate hypothesis in Phrynosomatid lizards. Evolution 67, 2614-2630 (2013).

441 4. Shine, R. A new hypothesis for the evolution of viviparity in reptiles. The American

$442 \quad$ Naturalist 145, 809-823 (1995).

443 5. Warner, D. A. \& Shine, R. Fitness of juvenile lizards depends on seasonal timing of 444 hatching, not offspring body size. Oecologia 154, 65-73 (2007).

445 6. Le Henanff, M., Meylan, S. \& Lourdais, O. The sooner the better: reproductive phenology

446 drives ontogenetic trajectories in a temperate squamate (Podarcis muralis). Biol J Linn

$447 \quad$ Soc 108, 384-395 (2013).

448 7. Ma, L., Buckley, L. B., Huey, R. B. \& Du, W.-G. A global test of the cold-climate

449 hypothesis for the evolution of viviparity of squamate reptiles. Global Ecology and

$450 \quad$ Biogeography 27, 679-689 (2018).

451 8. Beuchat, C. A. Temperature effects during gestation in a viviparous lizard. Journal of

$452 \quad$ Thermal Biology 13, 135-142 (1988).

453 9. Monasterio, C., Shoo, L. P., Salvador, A., Iraeta, P. \& Díaz, J. A. High temperature

454 constrains reproductive success in a temperate lizard: implications for distribution range

455 limits and the impacts of climate change. Journal of Zoology 291, 136-145 (2013).

456 10. Sanger, T. J., Kyrkos, J., Lachance, D. J., Czesny, B. \& Stroud, J. T. The effects of

457 thermal stress on the early development of the lizard Anolis sagrei. Journal of 

(2018). Royal Society B: Biological Sciences 286, 20192078 (2019).

463 12. Andrews, R. M., Mathies, T. \& Warner, D. A. Effect of Incubation Temperature on 464 Morphology, Growth, and Survival of Juvenile Sceloporus undulatus. Herpetological $465 \quad$ Monographs 14, 420-431 (2000).

466 13. Blackburn, D. G. Squamate Reptiles as Model Organisms for the Evolution of Viviparity. 467 Herpetological Monographs 20, 131-146 (2006).

468 14. Blackburn, D. G. Evolution of viviparity in squamate reptiles: Reversibility reconsidered. $469 \quad J$ Exp Zool B Mol Dev Evol 324, 473-486 (2015).

470 15. Molina-Venegas, R. et al. Assessing among-lineage variability in phylogenetic 471 imputation of functional trait datasets. Ecography 41, 1740-1749 (2018).

472 16. Noble, D. W. A., Stenhouse, V. \& Schwanz, L. E. Developmental temperatures and 473 phenotypic plasticity in reptiles: a systematic review and meta-analysis. Biological $474 \quad$ Reviews 93, 72-97 (2018).

475 17. Watson, C. M., Makowsky, R. \& Bagley, J. C. Reproductive mode evolution in lizards 476 revisited: updated analyses examining geographic, climatic and phylogenetic effects 477 support the cold-climate hypothesis. Journal of Evolutionary Biology 27, 2767-2780 $478 \quad$ (2014).

479 18. Beuchat, C. A. Reproductive Influences on the Thermoregulatory Behavior of a Live$480 \quad$ Bearing Lizard. Copeia 1986, 971-979 (1986).

481 19. Andrews, R. M. \& Mathies, T. Natural History of Reptilian Development: Constraints on 482 the Evolution of Viviparity. BioScience 50, 227-238 (2000). 
483 20. Andrews, R. M. Evolution of viviparity in squamate reptiles (Sceloporus spp.): a variant

484 of the cold-climate model. Journal of Zoology 250, 243-253 (2000).

485 21. Uller, T. \& Olsson, M. Offspring size and timing of hatching determine survival and 486 reproductive output in a lizard. Oecologia 162, 663-671 (2010).

487 22. Noble, D. W. A. et al. A comprehensive database of thermal developmental plasticity in $488 \quad$ reptiles. Sci Data 5, 1-7 (2018).

489 23. Borenstein, M., Hedges, L. V., Higgins, J. P. \& Rothstein, H. R. Effect Sizes Based on 490 Means. in Introduction to Meta-Analysis 21-32 (John Wiley \& Sons, Ltd, 2009). doi:10.1002/9780470743386.ch4.

24. Recknagel, H., Kamenos, N. A. \& Elmer, K. R. Common lizards break Dollo's law of irreversibility: Genome-wide phylogenomics support a single origin of viviparity and reevolution of oviparity. Molecular Phylogenetics and Evolution 127, 579-588 (2018).

25. R Core Team. R: A language and environment for statistical computing. R Foundation for Statistical Computing, Vienna, Austria. (2020).

26. Modern Phylogenetic Comparative Methods and Their Application in Evolutionary 2.

500 27. Paradis, E. \& Schliep, K. ape 5.0: an environment for modern phylogenetics and 501 evolutionary analyses in R. Bioinformatics 35, 526-528 (2019).

502 28. Pinheiro, J., Bates, D., DebRoy, S., Sarkar, D. \& Team, R. C. nlme: Linear and nonlinear $503 \quad$ mixed effects models. $R$ package version 3, 111 (2013).

504 29. Paradis, E. Analysis of Phylogenetics and Evolution with R. (Springer-Verlag, 2012). 505 doi:10.1007/978-1-4614-1743-9.

506 30. Hansen, T. F. Stabilizing selection and the comparative analysis of adaptation. Evolution $507 \quad 51,1341-1351(1997)$. 
508 31. Hadfield, J. D. MCMC Methods for Multi-Response Generalized Linear Mixed Models:

509 The MCMCglmm R Package. Journal of Statistical Software 33, 1-22 (2010).

510 32. Zheng, Y. \& Wiens, J. J. Combining phylogenomic and supermatrix approaches, and a

511 time-calibrated phylogeny for squamate reptiles (lizards and snakes) based on 52 genes

512 and 4162 species. Molecular Phylogenetics and Evolution 94, 537-547 (2016).

513 33. Nakagawa, S., Johnson, P. C. D. \& Schielzeth, H. The coefficient of determination R2

514 and intra-class correlation coefficient from generalized linear mixed-effects models

515 revisited and expanded. Journal of The Royal Society Interface 14, 20170213 (2017).

516 34. Orme, D. et al. CAPER: comparative analyses of phylogenetics and evolution in R.

517 Methods in Ecology and Evolution 3, 145-151 (2013).

518 35. Freckleton, R. P., Harvey, P. H. \& Pagel, M. Phylogenetic analysis and comparative data:

519 a test and review of evidence. Am. Nat. 160, 712-726 (2002).

520 36. Revell, L. J. phytools: an R package for phylogenetic comparative biology (and other

521 things). Methods in Ecology and Evolution 3, 217-223 (2012).

522 37. Blomberg, S. P., Garland, T. \& Ives, A. R. Testing for Phylogenetic Signal in

523 Comparative Data: Behavioral Traits Are More Labile. Evolution 57, 717-745 (2003).

524 38. Buuren, S. van \& Groothuis-Oudshoorn, K. mice: Multivariate Imputation by Chained

525 Equations in R. Journal of Statistical Software 45, 1-67 (2011).

526 39. Brooks, S. P. \& Gelman, A. General methods for monitoring convergence of iterative

527 simulations. Journal of computational and graphical statistics 7, 434-455 (1998).

528 40. Bollback, J. P. SIMMAP: Stochastic character mapping of discrete traits on phylogenies.

$529 \quad$ BMC Bioinformatics 7, 88 (2006).

530 41. Halliwell, B., Uller, T., Holland, B. R. \& While, G. M. Live bearing promotes the

531 evolution of sociality in reptiles. Nature Communications 8, 2030 (2017). 


\section{Figures}

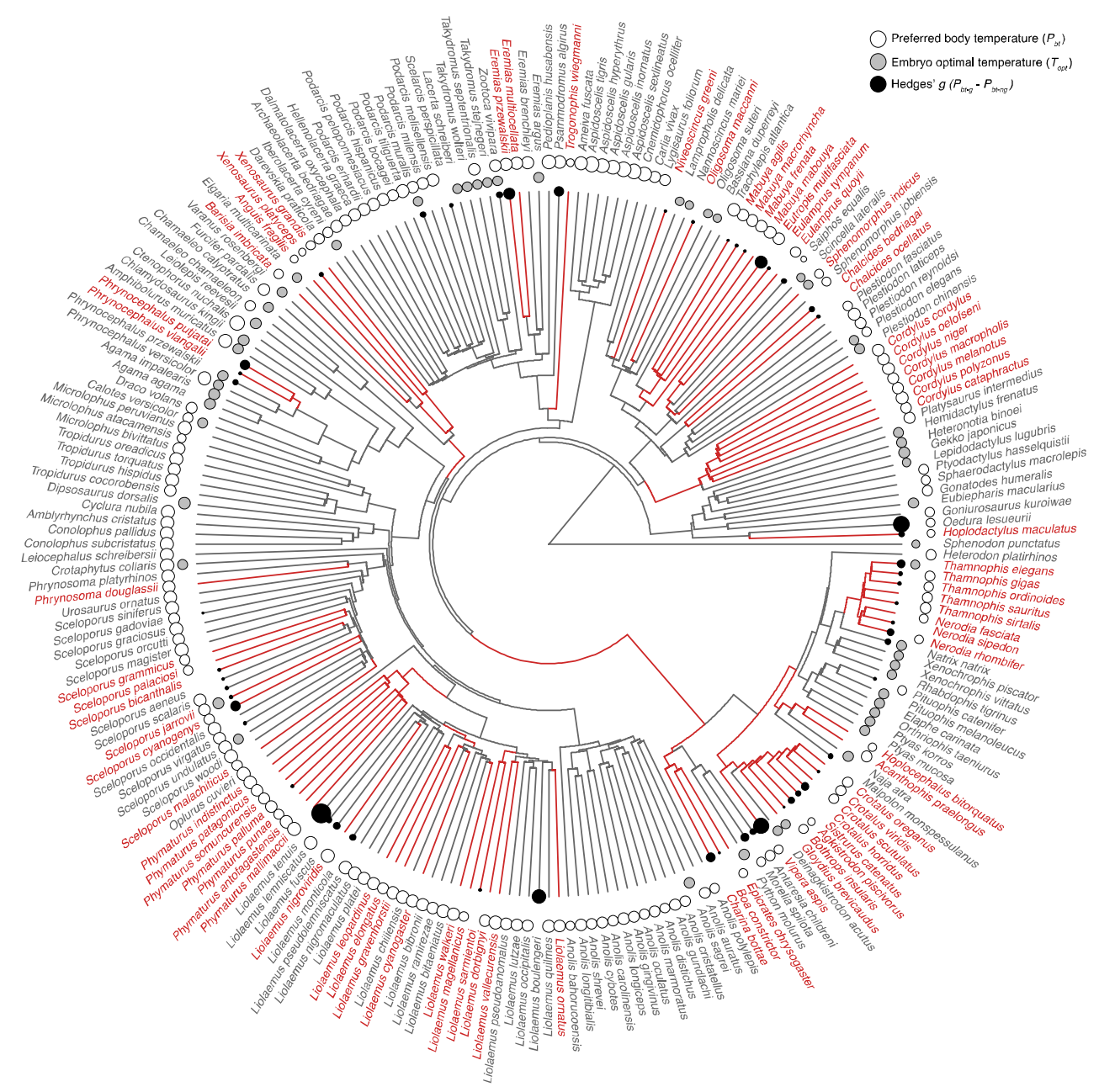

536 Figure 1. Variation in reproductive mode, preferred body temperature and plasticity in

537 female thermo-regulatory behaviour across 224 species of squamate reptile (lizards and

538 snakes). Tips and branches are coloured according to reproductive modes (red = live

539 bearing/viviparous, grey = egg laying/oviparous; branch colours represent predicted ancestral

540 values; Table S10). The size of white circles corresponds to the preferred body temperature

541 of female squamates ( $P_{b t}$ dataset, 163 species), the size of grey circles to the optimal

542 temperature for embryo survival in oviparous species ( $T_{\text {opt }}$ dataset, 51 species), and the size

543 of the black circles to the discrepancy between preferred body temperature in gravid versus

544 non-gravid females (Hedges' $g$ dataset; absolute values, 52 species). 


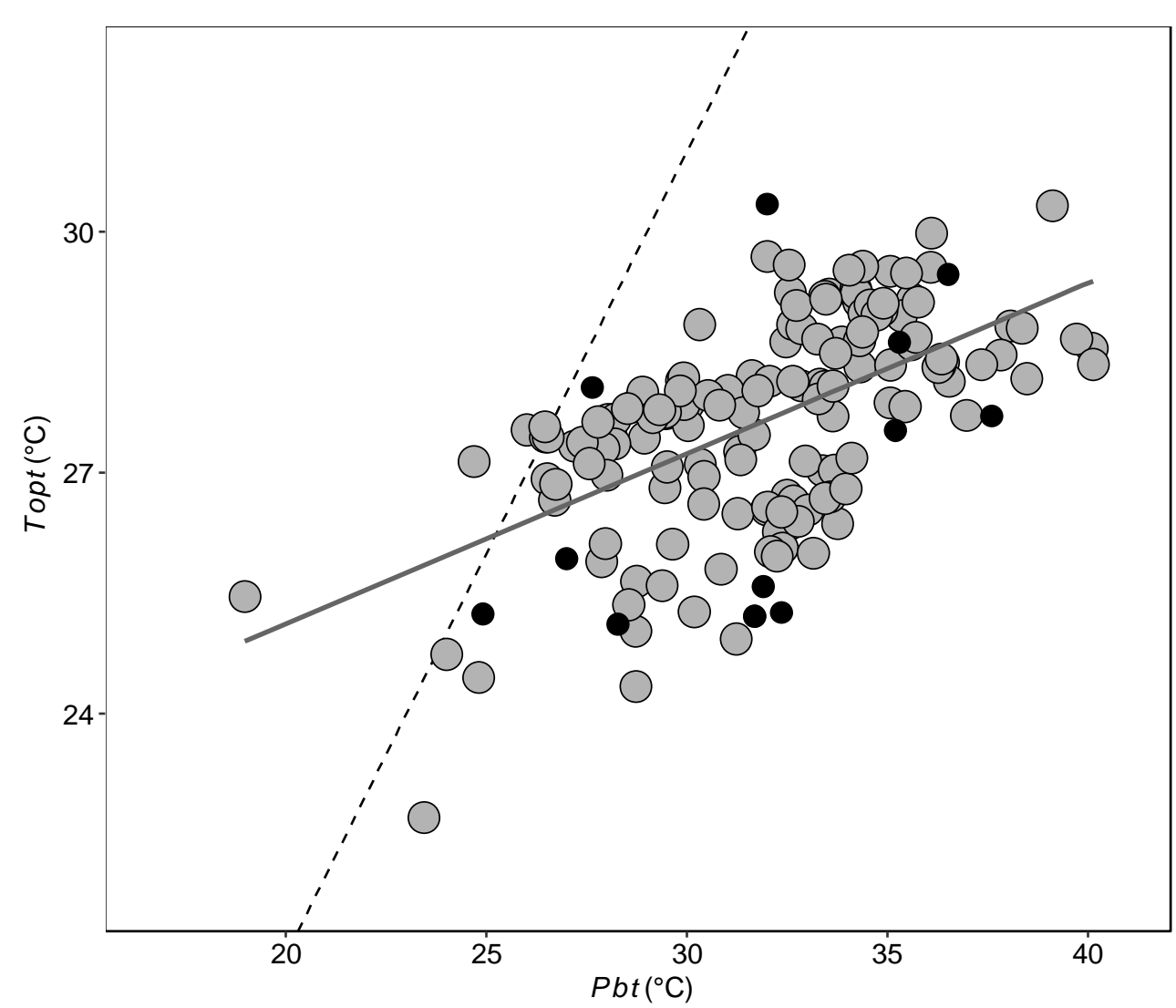

545

546 Figure 2. Mismatches in the thermal optima of mothers and embryos across 147

547 oviparous species of lizards and snakes. The relationship between female preferred body

548 temperature $\left(P_{b t}\right)$ and the temperature that maximizes hatching success of embryos $\left(T_{o p t}\right)$

549 predicted by a multi-response Bayesian Phylogenetic Mixed Model across egg laying species

550 (grey data points, regression lines $\pm 95 \%$ confidence intervals are plotted). Raw data for 12

551 species with both Pbt and Topt data shown by black data points. Despite high phylogenetic

552 signature in $P_{b t}$ and $T_{o p t}$, there was only weak evidence for co-evolution between the two

553 traits (Table S4). Deviations from the dotted line (1:1 relationship) indicate the magnitude of

554 the mismatch between adult and embryo thermal optima. Corresponding measurements of

$555 T_{\text {opt }}$ and $P_{b t}$ were not available for all oviparous species (total $\mathrm{n}_{\text {oviparous }}=147, \mathrm{n}_{\text {Pbt }}=103 ; \mathrm{n}_{\text {Topt }}$

$556=47 ; \mathrm{n}_{\mathrm{Pbt}} \& \mathrm{n}_{\text {Topt }}=10$ ), hence model predictions rather than raw data are shown (for plot of

557 raw data as well as correspondence between predicted and raw data values see Figure S2). 


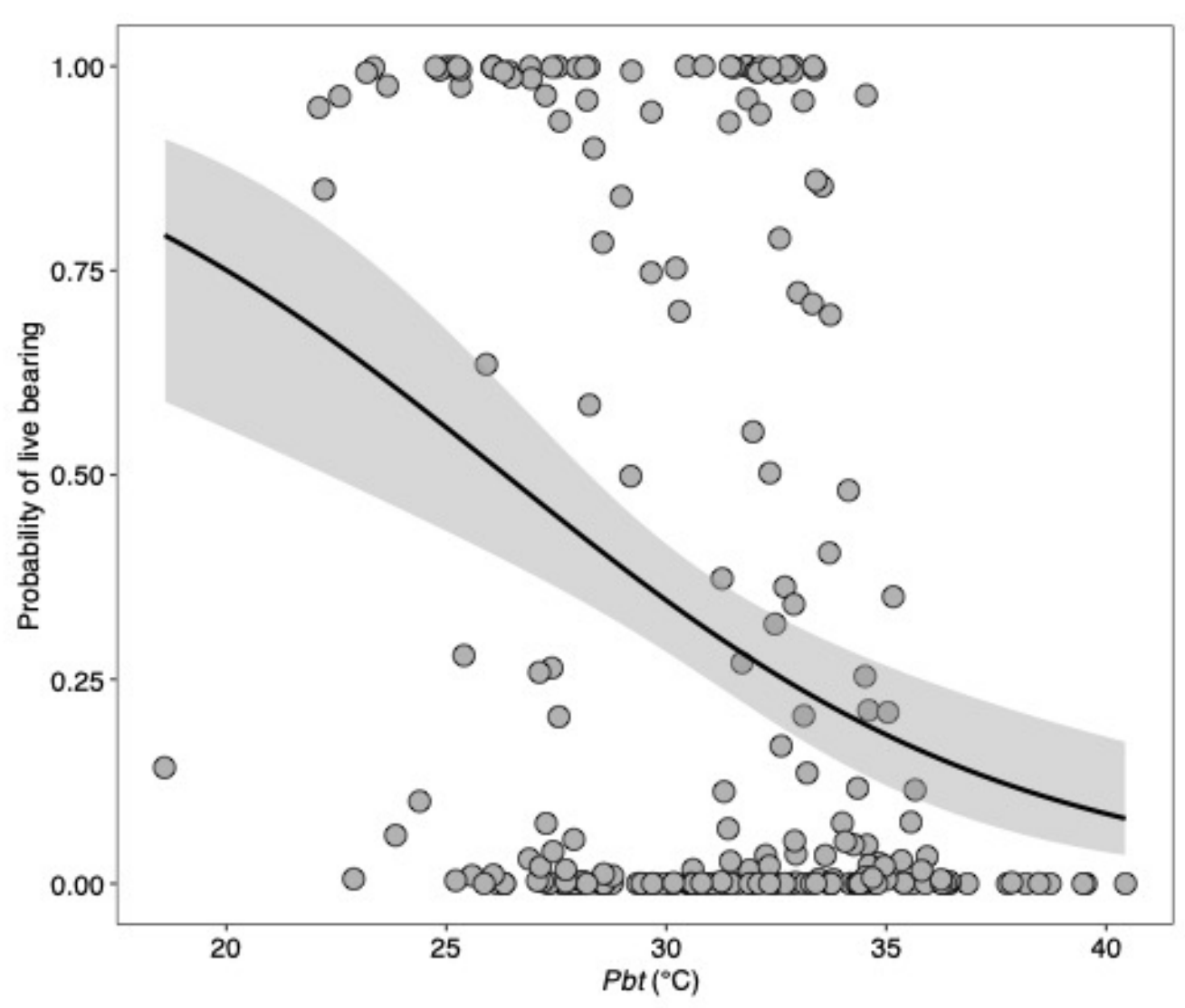

559 Figure 3. Probability of being a live-bearing species in relation to female non-gravid

560 preferred body temperature $\left(\mathbf{P}_{\mathbf{b t}}\right)$. The line and shading represent a logistic regression

561 curve $\pm 95 \%$ confidence interval. $\mathrm{P}_{\mathrm{bt}}$ was obtained for 103 oviparous and 61 viviparous

562 species. 
A

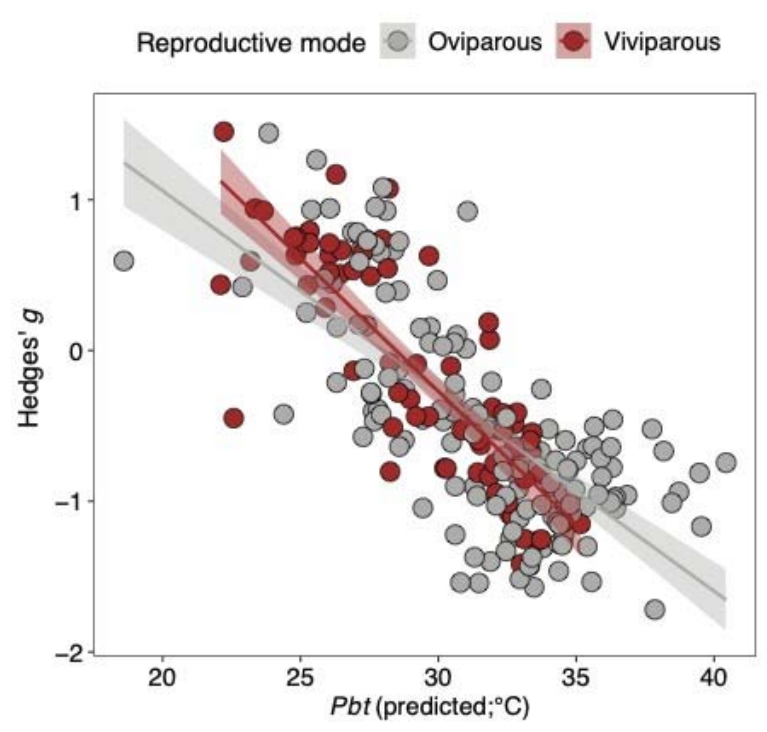

B No mismatch Topt $<\mathrm{Pbt}$

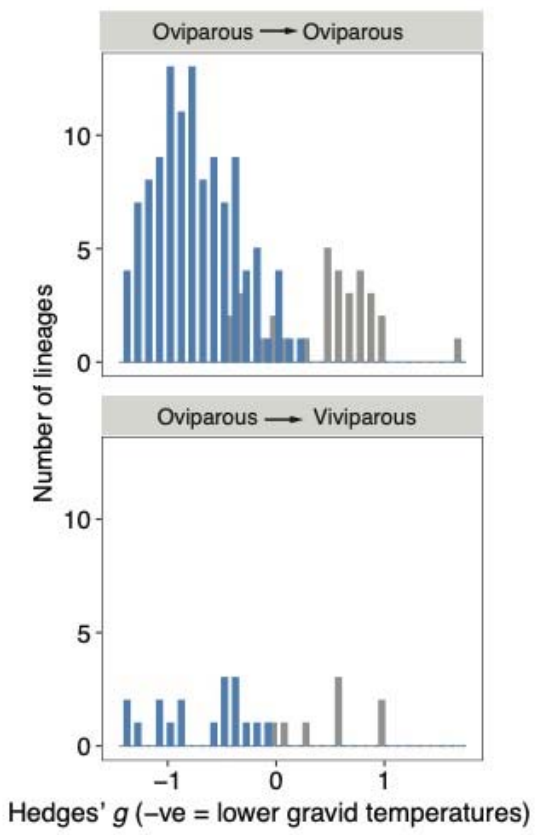

565

566 Figure 4. Female behavioural plasticity resolves the constraints imposed by thermal

567 physiology, such that transitions to live-bearing (viviparity) occur despite mismatches

568 between females and embryos. (A) Adjustment of body temperature in gravid females in

569 relation to their non-gravid preferred body temperature $\left(P_{b t}\right)$ across 52 extant oviparous and

570 viviparous lizards and snakes (Hedges' $g ; P_{b t-g}-P_{b t-n g}$ ). Regression lines $\pm 95 \%$ credible

571 intervals are plotted. High values of Hedges' $g$ signify an increase in body temperature when

572 gravid while low values imply a decrease. (B) The adjustment of body temperature by

573 females in lineages that remained egg laying (top panel) and lineages that evolved live

574 bearing (bottom panel) according to the presence or absence of a thermal mismatch between

575 females and their offspring (inferred from Hedges' $g$ data shown in panel A). 